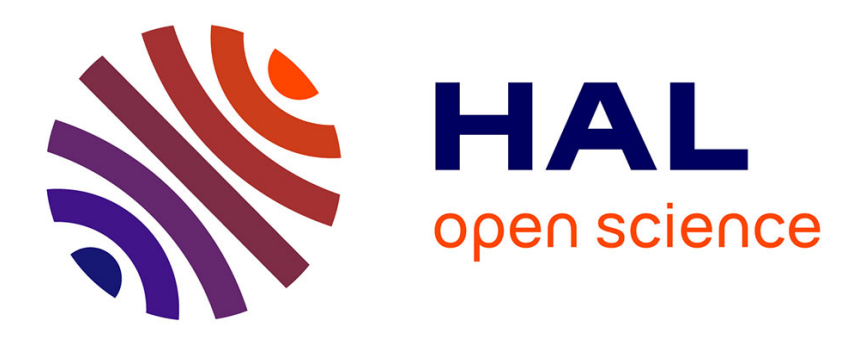

\title{
Sensing Multifrequency THz-waves with a Photomixer
} Florin Lucian Constantin

\section{To cite this version:}

Florin Lucian Constantin. Sensing Multifrequency THz-waves with a Photomixer. Imaging Systems and Applications 2020, 2020, Vancouver, Canada. pp.1-2, 10.1364/3D.2020.JW5C.5 . hal-03054317

\section{HAL Id: hal-03054317 https://hal.science/hal-03054317}

Submitted on 11 Dec 2020

HAL is a multi-disciplinary open access archive for the deposit and dissemination of scientific research documents, whether they are published or not. The documents may come from teaching and research institutions in France or abroad, or from public or private research centers.
L'archive ouverte pluridisciplinaire HAL, est destinée au dépôt et à la diffusion de documents scientifiques de niveau recherche, publiés ou non, émanant des établissements d'enseignement et de recherche français ou étrangers, des laboratoires publics ou privés.

$$
\text { Copyright }
$$




\title{
Sensing Multifrequency THz-waves with a Photomixer Florin Lucian Constantin \\ Laboratoire PhLAM, CNRS UMR 8523, 59655 Villeneuve d'Ascq, France \\ FL.Constantin@univ-lille1.fr
}

\begin{abstract}
The nonlinear electrical response from a low temperature grown GaAs photomixer driven with the optical beat of two continuous-wave lasers is exploited for frequency comb generation and phase-coherent THz frequency comb heterodyne detection. (C) 2020 The Author(s)
\end{abstract}

\section{Introduction}

The terahertz-waves are useful for several applications including biological imaging, remote sensing, high-speed telecommunications, and security screening [1]. The optical frequency down-conversion with a photoconductive antenna (photomixing) was exploited for continuous-wave (cw) THz generation [2] by driving an antenna with the photocurrent generated by beating two $\mathrm{cw}$ lasers in an ultrafast photoconductor. Compact, room-temperature operated, cost-effective and low-power consuming THz photomixing systems with optical frequency control enabled narrow spectral linewidth, wide frequency tunability, high dynamic range and high signal-to-noise ratio. Here, the electrical nonlinear response of a low temperature grown GaAs (LTG-GaAs) photomixer is exploited for frequency comb generation and detection. Multifrequency photonic generation is demonstrated by photomixing with a modulated bias voltage [3]. Alternatively, a THz frequency comb is generated by driving a solid-state electronic frequency multiplier with a pulsed microwave synthesizer [4]. The heterodyne detection with the photomixer exploits the optical beat as local oscillator to down-convert the THz frequency comb to the microwave domain $[4,5]$.

\section{Results}

The photomixing model developed here is based on uniform unidimensional carrier transport in a thin LTG-GaAs layer. Two spatially superposed coherent optical waves, detuned by an angular frequency $\omega$ and carrying the same optical power $\mathrm{P}$ enter at normal incidence in the photoconductor and generate a current density expressed as:

$$
j(t)=\frac{2 e \eta P}{\hbar \bar{\omega} A d}\left[\tau_{n}(E) v_{n}(E)\left(1+\frac{\omega \tau_{n}(E) \sin (\omega t)+\cos (\omega t)}{1+\left(\omega \tau_{n}(E)\right)^{2}}\right)+\tau_{p}(E) v_{p}(E)\left(1+\frac{\omega \tau_{p}(E) \sin (\omega t)+\cos (\omega t)}{1+\left(\omega \tau_{p}(E)\right)^{2}}\right)\right]
$$

The laser beams, carrying nearly the same energy $\hbar \varpi$, make an optical beat that is focused on a photoconductive area $\mathrm{A}$, and absorbed within a depth $\mathrm{d}$ with a quantum efficiency $\eta$. The dependences of the carrier lifetimes $\tau_{\mathrm{n}, \mathrm{p}}(E)$ with the electric field [6] are described here with a linear electric field dependence arising from carrier heating :

$$
\tau_{n, p}(E) \approx \tau_{0 n, 0 p}\left[1+3 \lambda e E /\left(2 k_{B} T\right)\right]
$$

in function of the Boltzmann constant $k_{B}$, the electron charge $e$, the room temperature $T=300 \mathrm{~K}$, the low-field carrier lifetimes $\tau_{0 n, 0 p}$ and the distance $\lambda$ between Coulomb barriers in LTG-GaAs. The carrier drift velocities have a nonlinear dependence on the electrical field $[7,8]$, expressed as :

$$
v_{n, p}(E)=v_{s n, s p} /\left[1+v_{s n, s p} /\left(\mu_{0 n, 0 p} E\right)\right]
$$

with the saturation velocities $v_{s n, s p}$ and the low-field mobilities $\mu_{0 n, 0 p}$ of the electrons and holes. The electric field in the photoconductor is expressed in the parallel-plate approximation as an alternative electric field (amplitude $E_{A C}=V_{A C} / r$, angular frequency $\omega_{A C}$, electrode spacing $\left.r\right)$ superposed to a bias electric field $\left(E_{D C}=V_{D C} / r\right)$ :

$$
E(t)=V_{D C} / r+\left(V_{A C} / r\right) \times \cos \left(\omega_{A C} t\right)
$$

The electric field is applied to the photomixer electrodes either by modulating the bias voltage or by an incoming $\mathrm{THz}$ radiation received by the antenna. The electrical nonlinearity arising from $v_{n, p}(E)$ and $\tau_{n, p}(E)$ dependences yields a current density with spectral components at angular frequencies $\omega \pm n \omega_{A C}$ (n integer). The amplitudes and phases of the spectral components of the frequency comb are analytically calculated with eq. (1-4) for a given $E_{D C}$ using a power series expansion in terms of $E_{A C}$. Particularly, phase-coherent $\mathrm{THz}$ frequency comb down-conversion may be performed by heterodyning a multifrequency THz-wave with an optical beat exploited as local oscillator (LO). 
The experiment (Fig. 1.A) is based on a LTG-GaAs photomixer with a log-spiral antenna driven by the optical beat of two extended cavity diode lasers emitting at $820 \mathrm{~nm}$. The extremities of the antenna are connected to a coaxial microwave line addressed with a bias- $T$, which is used for coupling the modulation signal and the bias voltage to the photomixer and to measure its response with a microwave spectrum analyser. A $75-110 \mathrm{GHz}$ electronic frequency multiplier driven by a microwave synthesizer is used to generate a THz-wave which is focused on the photomixer antenna with a silicon lens. On one hand, the modulation of the photomixing current is observed by coupling a radiofrequency signal to the photomixer driven by an optical beat in the microwave domain. The spectrum of the photomixing signal (Fig. 1.B) displays the carrier and sidebands spaced by the modulation frequency. The amplitudes of the spectral components are calculated with eq. (1-4) using the modulation parameters, the LTG-GaAs photoconductor parameters from refs. [6-8], and the photomixer parameters indicated below. The results are multiplied with a common factor in order to match the amplitudes of the most intense spectral components and plotted with bars. The calculated and measured amplitudes agree for $\omega \pm 2 \omega_{A C}$ sidebands but not for the others. The model may be improved by taking into account spatial dependence of the current density, optical power and spatial charge separation effects in the photoconductor. On the other hand, the photomixer is exploited for heterodyne detection of the THz-waves generated with a frequency multiplier driven by a pulse-modulated microwave synthesizer (Fig. 1.C). A monochromatic THz-wave is down-converted to $\sim 1.1 \mathrm{GHz}$, and recorded with a signal-tonoise ratio of 10 in $1 \mathrm{MHz}$ bandwidth. The responsivity is at the $10^{-3} \mathrm{~A} / \mathrm{W}$ level. The linewidth of the signal is related to the spectral purity of the free-running diode lasers. When the microwave synthesizer is pulse-modulated, the spectral components of the resulting $\mathrm{THz}$ frequency comb are down-converted by the heterodyne detection scheme and individually resolved. The heterodyne detection signal is sensitive to the THz-wave amplitude and phase and, via antenna's geometry, to the THz-wave spatial mode and polarization state. The photomixing approach with an ultrafast photoconductor enables integration of the optical beat generation with the electrically-driven optoelectronic frequency conversion in a single photonic circuit for $\mathrm{THz}$ frequency comb generation and detection.
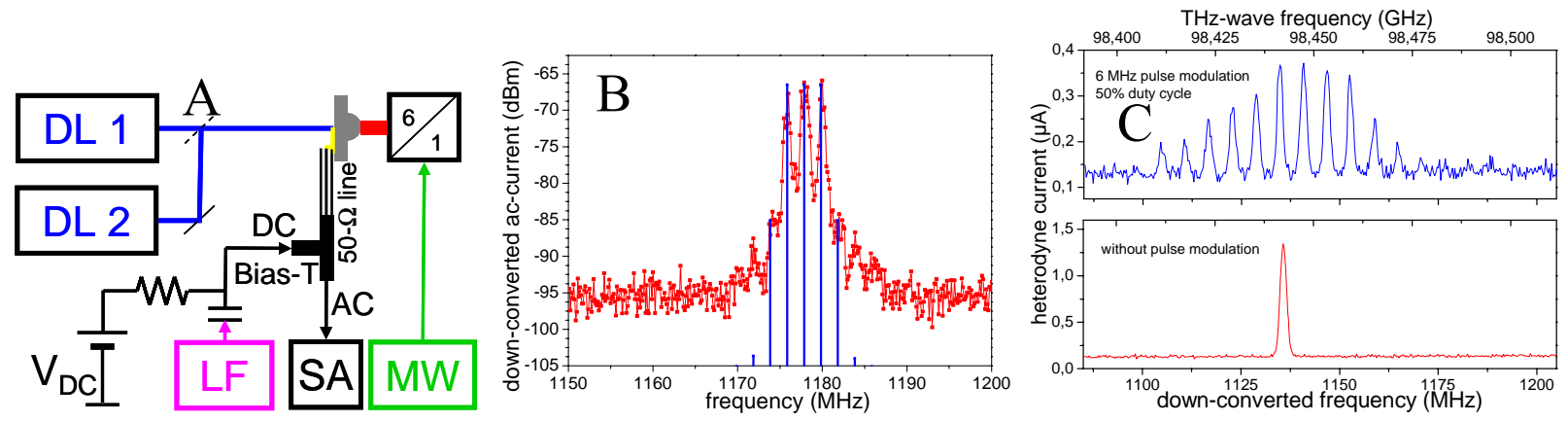

Fig. 1. A. The experimental setup. DL1, DL2 diode lasers, SA spectrum analyzer, LF radiofrequency synthesizer, MW microwave synthesizer. B. Spectrum of a modulated photomixing signal. Optical power $2 \times 20 \mathrm{~mW}$, optical beat at $\omega=2 \pi \times$ $1178 \mathrm{MHz}$. Modulation voltage $V_{A C}=0.5 \mathrm{~V}$ at $\omega_{A C}=2 \pi \times 2 \mathrm{MHz}$, bias voltage $\mathrm{V}_{D C}=0.517 \mathrm{~V}$. The calculated amplitudes of the spectral components are plotted with blue bars. Photomixer parameters used in the calculations: $\mathrm{r}=2 \mu \mathrm{m}, \mathrm{d}=2 \mu \mathrm{m}, \mathrm{A}=12.6 \mu \mathrm{m}^{2}$, $\hbar \varpi / e=1.5 \mathrm{~V}, \eta=0.68$. RBW=300 kHz, sweep time $4 \mathrm{~ms}, 2$ video averages. C. Spectrum of the heterodyne detection signal of a

THz-wave at $\omega_{A C}=2 \pi \times 98.4 \mathrm{GHz}$ (lower panel, red line). Down-converted THz frequency comb generated with pulse modulation at $6 \mathrm{MHz}$ with $50 \%$ duty cycle (upper panel, blue line). Bias voltage $\mathrm{V}_{D C}=85 \mathrm{mV}$. Optical beat power $2 \times 20 \mathrm{~mW}$. $\mathrm{RBW}=1 \mathrm{MHz}$, sweep time $4 \mathrm{~ms}, 16$ video averages.

\section{References}

[1] S.S. Dhillon et al., "The 2017 terahertz science and technology roadmap," J. Phys. D 50, 043001 (2017).

[2] E.R. Brown et al., "Photomixing up to 3.8 THz in low-temperature-grown GaAs," Appl. Phys. Lett. 66, 285-287 (1995).

[3] F.L. Constantin, "Direct-current modulation of a photomixing signal," in Nonlinear Optics and its Applications IV, B.J. Eggleton, N.G.R. Broderick and A.L. Gaeta, eds., Proc. of SPIE Vol. 9894 (Society Of Photo-Optical Instrumentation Engineers, Bellingham, WA, 2016), pp. 98941K.

[4] F.L. Constantin, "Photomixing for coherent retrieval of THz waveforms from a frequency multiplier," EPJ Web of Conferences $\mathbf{4 1}, 09016$ (2013).

[5] F.L. Constantin, "Phase-coherent heterodyne detection in the terahertz regime with a photomixer," IEEE J. Quantum Electr. 47, 1458-1462 (2011).

[6] N. Zamdmer and Q. Hu, "Increase in response time of low-temperature-grown GaAs photoconductive switches at high voltage bias," Appl. Phys. Lett. 75, 2313-2315 (1999).

[7] E.R. Brown, “A photoconductive model for superior GaAs THz photomixer,” Appl. Phys. Lett. 75, 769-771 (1999).

[8] E.R. Brown et al., "Milliwatt output levels and superquadratic bias dependence in a low-temperature-grown GaAs photomixer," Appl. Phys.

Lett. 64, 3311-3313 (1994). 